\title{
EVIDENCIA Y RECOMENDACIÓN. ¿LA NEUROMONITORIZACIÓN INTERMITENTE ES ÚTIL PARA LA REDUCCIÓN DE PARÁLISIS DE NERVIO LARÍNGEO RECURRENTE EN CIRUGÍA DE TIROIDES?
}

\section{Evidence and recommendation. Intermitent neuromonitoring in thyroid surgery is usefulness for reduction of recurrent nerve palsy?}

\author{
José Luis PARDAL-REFOYO \\ SACYL. Complejo Asistencial de Zamora. Servicio de Otorrinolaringología. Zamora. España. \\ Correspondencia: jlpardal@usal.es
}

Fecha de recepción: 18 de julio de 2016

Fecha de aceptación: 1 de septiembre de 2016

Fecha de publicación: 4 de septiembre de 2016

Fecha de publicación del fascículo: 1 de marzo de 2017

Conflicto de intereses: Los autores declaran no tener conflictos de intereses Imágenes: Los autores declaran haber obtenido las imágenes con el permiso de los pacientes Política de derechos y autoarchivo: se permite el autoarchivo de la versión post-print (SHERPA/RoMEO) Licencia CC BY-NC-ND. Licencia Creative Commons Atribución-NoComercial-SinDerivar 4.0 Internacional (C) Universidad de Salamanca. Su comercialización está sujeta al permiso del editor ted to thyroidectomy [intervention], the use of neuromonitoring against the single visual identification of recurrent laryngeal nerve (RLN) [comparison], offers advantages? [result]. Material and Methods: Literature review in PubMed, Scopus and Cochrane Library data descriptors and 
search strategy: $(((((($ laryngeal) OR larynx $))$ AND nerve) AND monitoring) AND thyroidectomy)) AND meta-analysis. 10 items in English or Spanish of which 7 were selected for the qualitative study were obtained. Results: Level of evidence. Evidence for the lower incidence of transient unilateral paralysis in patients with neuromonitoring is moderate-high. Evidence on the lower incidence of permanent unilateral paralysis of RLN with NM is low. Evidence on the lower incidence of bilateral paralysis of RLN with neuromonitoring is low. Conclusions: Recommendation. Concerning the reduction of transient paralysis of the RLN the recommendation of the use of neuromonitoring in thyroid surgery is strongly in favor. Regarding permanent paralysis recommendation is weak in favor (no recommendation against). Regarding prevention of bilateral laryngeal paralysis recommendation for weak. The decision to use NM cannot be based on the incidence of RLN paralysis.

KEYWORDS

thyroidectomy; recurrent laryngeal nerve; neuromonitoring

\section{SITUACIÓN DEL TEMA}

La aplicación de la neuromonitorización (NM) a la cirugía de tiroides fue descrita por Riddell [1] que basaba la preservación de la función del nervio laríngeo recurrente (NLR) en su identificación visual, en la comprobación funcional tras finalizar la intervención mediante estímulo eléctrico y en evaluar la motilidad laríngea mediante laringoscopia de rutina pre y postoperatoria. Estos principios se mantienen actualmente.

El estímulo puede ser intermitente (el más empleado) o continuo (con electrodo fijo en el nervio vago); ambas técnicas son compatibles y pueden realizarse simultáneamente.

Los electrodos de registro pueden ser de superficie adheridos al tubo endotraqueal (TET) en contacto con las cuerdas vocales o de aguja insertados en los músculos tiroaritenoideos a través de la membrana cricotiroidea (transligamentario o translaríngeo - TL-).

Tres son las preguntas más debatidas en la literatura sobre la NM en la cirugía de tiroides: su capacidad para ayudar a identificar el NLR, su precisión para establecer el estado funcional del nervio al concluir la cirugía y su utilidad en la reducción de parálisis de NLR. La respuesta a cada una de ellas debe hacerse investigando por separado.

En una revisión realizada sobre publicaciones del periodo 2002-2013 que indicaban la precisión de la neuromonitorización en cirugía de tiroides se observó una gran variabilidad entre los investigadores respecto a los datos de sensibilidad, especificidad, valor predictivo positivo y valor predictivo negativo [2], siendo difícil de comparar unos con otros debido a la inclusión de patologías y técnicas quirúrgicas diversas, a la aplicación de diversas técnicas de neuromonitorización, a la variabilidad en el momento de realizar la laringoscopia, a la inclusión de tipo de parálisis transitorias y permanentes a veces no bien especificadas, al existir diversos tipos de diseño de estudio y al año de publicación que implica técnicas y tecnologías diferentes difícilmente comparables. No obstante, la precisión de la NM como prueba para establecer el estado funcional del NLR es alta.

La precisión de la prueba se ve afectada por el tipo de registro (tipo de electrodo - TET o TL, palpación - twitch -), el punto de aplicación del electrodo de estimulación (sobre el nervio directamente o en su cercanía), el tipo de estímulo aplicado (intermitente o continuo), el nervio estimulado y registro (NLR -registro $\mathrm{R}$ - o nervio vago - registro $\mathrm{V}-$ ) y la secuencia de registros (en dos pasos - V1 inicial y V2 finalo en cuatro pasos - V1, R1, R2, V2-) [2].

A estos condicionantes pueden añadirse otros muchos que pueden influir en la obtención del registro como es la experiencia del cirujano en la utilización de la NM e interpretación de la señal.

Hay dos situaciones importantes en la interpretación de la señal: la situación falso negativo _-infrecuente pero peligrosa dado que significa la presentación de parálisis de NLR con una señal previa normal-y la situación, más frecuente, de falso positivo - de menor riesgo para el paciente, pero de conflicto para el cirujano ya que puede condicionar la técnica debido a la ausencia o pérdida de señal-. La ausencia o pérdida de la señal pueden ser debidas a una técnica incorrecta - programación de parámetros-, a malposición de los electrodos de registro o que haya sangre 0 sustancias lubricantes que no permiten el contacto correcto de los electrodos de registro con la superficie de la cuerda vocal -en electrodos de superficie-, problemas en el cableado - 
fallos de conexión-, a interferencias -estimulador transcutáneo, marcapasos, electrobisturí, enredo de cables- $-[2,3]$.

Los argumentos en contra del uso de la NM en cirugía tiroidea que se proponen son, entre otros, que la incidencia de parálisis permanente no se reduce, que los falsos positivos interfieren en el desarrollo normal de la cirugía, que los falsos negativos suponen riesgo para el paciente, que la baja incidencia de parálisis bilateral no justifica el uso de la NM, el coste del equipo, la falta de consenso, la heterogeneidad en su uso y que la integridad anatómica no implica integridad funcional [3].

Los argumentos a favor de utilizar la NM son que ayuda en la localización del NLR especialmente en cirugía dificultosa (reintervención, infiltración) y durante la disección del NLR (trayectos, ramificaciones), ayuda en la localización de los cabos del NLR en caso de sección, informa sobre la funcionalidad del NLR al finalizar la cirugía, puede informar sobre el grado de lesión del NLR, puede reducir la incidencia de parálisis del NLR, ayuda a tomar decisiones durante la cirugía en caso de pérdida de señal o parálisis contralateral por lo que puede aumentar la seguridad del paciente, aumenta la confianza del cirujano durante la cirugía, tiene utilidad durante la docencia facilitando el aprendizaje continuo y puede tener utilidad medicolegal [2].

La auténtica utilidad de la NM está en que informa sobre el estado funcional del NLR al finalizar la cirugía orientando sobre una posible parálisis que habrá que confirmar mediante laringoscopia (validez) [2]. La tasa de parálisis es una consecuencia de la cirugía que depende de variables no relacionadas con la neuromonitorización y puede ayudar en la prevención de la parálisis bilateral [1, 2].

Este artículo tiene como objetivo evaluar la utilidad de la neuromonitorización basada en la revisión bibliográfica y el nivel de evidencia. En la metodología se siguen las directrices para este tipo de artículos [4].

\section{PREGUNTA CLÍNICA (PICO)}

En un paciente con patología de tiroides [paciente], sometido a tiroidectomía [intervención], la utilización de neuromonitorización frente a la sola identificación visual del nervio laríngeo recurrente [comparación], ¿ofrece ventajas? [resultado].

\section{BÚSQUEDA BIBLIOGRÁFICA}

Se realizó una búsqueda bibliográfica en las bases de datos PubMed (http://www.ncbi.nlm.nih.gov/pubmed/advanced), Cochrane Library (http://onlinelibrary.wiley.com/cochranelibrary/search/) y Scopus (https://www.scopus.com) de los ensayos clínicos y metanálisis en idioma español e inglés a fecha 1 de mayo de 2016 que evalúan la utilización de neuromonitorización en tiroidectomía realizada mediante técnica abierta convencional frente a la sola identificación visual. Los descriptores y estrategias de búsqueda se resumen en la figura 1.

Se seleccionaron 10 metanálisis en la primera revisión, de los que se excluyeron tres artículos (uno porque trataba sobre cirugía robótica, otro porque era una carta al director y otro escrito en chino).

Búsqueda bibliográfica (PubMed, Scopus, Cochrane)
(resumen)
1- (((((laryngeal) OR larynx)) AND nerve) AND monitor-
ing) AND thyroidectomy: 350
2- $(((((((($ laryngeal) OR larynx)) AND nerve) AND moni-
toring) AND thyroidectomy)) AND trial): 34 (ECA)
3- (((((((laryngeal) OR larynx)) AND nerve) AND moni-
toring) AND thyroidectomy)) AND meta-analysis: 10
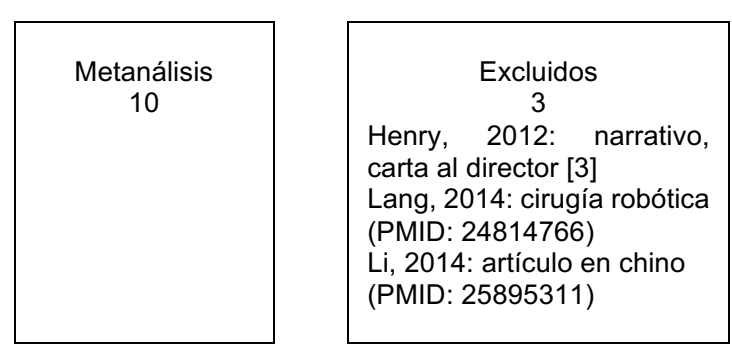

Incluidos en el
estudio cualitativo
$7[5-11]$

Figura 1. ¿La neuromonitorización intermitente es útil para la reducción de parálisis de nervio laríngeo recurrente en cirugía de tiroides? Diagrama con la estrategia de búsqueda.

En la tabla 1 se resumen las características más importantes de los estudios seleccionados. 


\section{¿LA NEUROMONITORIZACIÓN INTERMITENTE ES ÚTIL EN CIRUGÍA DE TIROIDES? PARDAL-REFOYO JL}

En los metanálisis seleccionados se evalúan los parámetros: coste, parálisis laríngea bilateral en tiroidectomía total, parálisis laríngea unilateral transitoria, parálisis laríngea unilateral permanente e identificación del NLR en tiroidectomía realizada con o sin NM.

La NM ofrece ventajas en la menor incidencia de parálisis laríngea respecto a la identifica- ción visual sola aunque no consiga significación estadística (o al menos no se ha demostrado desventaja).

Por otro lado, los resultados se basan en estudios observacionales no aleatorios por lo que se requieren ensayos multicéntricos aleatorizados.

Tabla 1. ¿La neuromonitorización intermitente es útil para la reducción de parálisis de nervio laríngeo recurrente en cirugía de tiroides? Tabla-resumen de los resultados de la búsqueda bibliográfica.

\begin{tabular}{|c|c|c|c|}
\hline Autor, año & $\begin{array}{l}\text { Tipo de estudio } \\
\text { (muestra) }\end{array}$ & Resultado principal & $\begin{array}{l}\text { Comentarios } \\
\text { (limitaciones) }\end{array}$ \\
\hline Sanabria, 2015 & Metanálisis. & $\begin{array}{l}\text { No diferencia significativas en } \\
\text { la incidencia de parálisis de } \\
\text { NLR sin NM (1\%)y con neuro- } \\
\text { monitorización }(1,6 \%) \text {. }\end{array}$ & $\begin{array}{l}\text { Estudio de costes. La cirugía } \\
\text { sin neuromonitorización es } \\
\text { menos cara. La NM de rutina } \\
\text { en la tiroidectomía total con } \\
\text { bajo riesgo de lesión del NLR } \\
\text { no es útil ni rentable en el sis- } \\
\text { tema de salud de Colombia. }\end{array}$ \\
\hline Pardal, 2015 & $\begin{array}{l}\text { Metanálisis. } \\
\text { Incluye estudios observacio- } \\
\text { nales. } \\
\text { Parálisis bilateral de NLR en } \\
\text { tiroidectomía total. } \\
40 \text { artículos. } \\
54 \text { series de casos (sin NM, } \\
25 \text {; con NM, 29) } 30922 \text { pa- } \\
\text { cientes. }\end{array}$ & $\begin{array}{l}\text { La prevalencia de parálisis de } \\
\text { NLR en las series con NM son } \\
\text { más bajas }(2.43 \% \text {, [1.55 a } \\
3.5 \% \text { ] frente a } 5.18 \% \text { [ } 2.53 \text { a } \\
8.7 \% \text { ] ) que equivale a una re- } \\
\text { ducción del riesgo del } 2.75 \% \text { o } \\
\text { con un número de casos a tra- } \\
\text { tar de } 364,13 \text {. el grupo con } \\
\text { NM es más homogéneo que } \\
\text { sin NM (I2= } 7,52 \% \text { frente } \\
\text { I2=79,32\%). }\end{array}$ & $\begin{array}{l}\text { Solo parálisis NLR bilateral. } \\
\text { El riesgo de parálisis laríngea } \\
\text { bilateral es menor en los estu- } \\
\text { dios con NM. } \\
\text { Las diferencias observadas } \\
\text { son muy imprecisas dado que } \\
\text { el número de parálisis larínga } \\
\text { bilateral observadas es muy } \\
\text { bajo. }\end{array}$ \\
\hline Rulli, 2014 & $\begin{array}{l}\text { Metanálisis. } \\
8 \text { artículos. } \\
5257 \text { NLR. }\end{array}$ & $\begin{array}{l}\text { Menor tasa de parálisis transi- } \\
\text { torias en las series con NM. }\end{array}$ & \\
\hline Pisanu, 2014 & $\begin{array}{l}\text { Metanálisis. } \\
20 \text { estudios. } \\
\text { Incluye estudios observacio- } \\
\text { nales. } \\
23512 \text { pacientes. } \\
35513 \text { NLR. }\end{array}$ & $\begin{array}{l}\text { La incidencia de parálisis con } \\
\text { NM fue } 3.47 \% \text { y en el grupo } \\
\text { sin NM de } 3.67 \% \text {. }\end{array}$ & $\begin{array}{l}\text { No hay diferencias significati- } \\
\text { vas en la tasa de parálisis } \\
\text { transitoria o permanente con o } \\
\text { sin uso de NM. }\end{array}$ \\
\hline Zheng, 2013 & $\begin{array}{l}\text { Metanálisis. } \\
5 \text { ECA y } 12 \text { ensayos compa- } \\
\text { rativos. } \\
36487 \text { NLR. }\end{array}$ & $\begin{array}{l}\text { Parálisis de NLR } 3.37 \% \text { con } \\
\text { NM frente a } 3.76 \% \text { sin NM. }\end{array}$ & $\begin{array}{l}\text { No hay diferencias significati- } \\
\text { vas en la presentación de pa- } \\
\text { rálisis. }\end{array}$ \\
\hline Sanabria, 2013 & $\begin{array}{l}\text { Metanálisis. } \\
6 \text { estudios. } \\
1.602 \text { pacientes. } \\
\text { 3.064 NLR. }\end{array}$ & $\begin{array}{l}\text { Reducción significativa en la } \\
\text { tasa de parálisis temporal de } \\
\text { NLR en el grupo con NM. } \\
\text { No reducción de la tasa de pa- } \\
\text { rálisis definitiva. }\end{array}$ & $\begin{array}{l}\text { Calidad metodológica de los } \\
\text { estudios baja o muy baja. }\end{array}$ \\
\hline Higgins, 2011 & $\begin{array}{l}\text { Metanálisis. } \\
1 \text { ECA, } 7 \text { ensayos comparati- } \\
\text { vos y } 34 \text { series de casos }\end{array}$ & $\begin{array}{l}\text { Incidencia de parálisis con } \\
\text { NM fue del } 3,52 \% \text { y sin NM del } \\
3,12 \% \text {. }\end{array}$ & $\begin{array}{l}\text { No hay diferencias significati- } \\
\text { vas en la tasa de parálisis de } \\
\text { NLR entre los grupos con o } \\
\text { sin NM. }\end{array}$ \\
\hline
\end{tabular}

NLR: nervio laríngeo recurrente; NM: neuromonitorización intraoperatoria 


\section{RESUMEN DE EVIDENCIA (GRADE)}

La evidencia sobre la menor incidencia de parálisis unilateral transitoria en pacientes con neuromonitorización es moderadaalta.

La evidencia sobre la menor incidencia de parálisis unilateral permanente de NLR con NM es baja.

La evidencia sobre la menor incidencia de parálisis bilateral de NLR con neuromonitorización es baja.

\section{RECOMENDACIONES (GRADE)}

Respecto a la reducción de la parálisis transitoria del NLR la recomendación de la utilización de la neuromonitorización en cirugía de tiroides es fuerte a favor.

Respecto a la parálisis permanente la recomendación es débil a favor (no hay recomendación en contra).

Respecto a la prevención de la parálisis laríngea bilateral la recomendación es débil a favor.

La decisión de utilizar NM no puede basarse exclusivamente en la incidencia de parálisis de NLR.

\section{BIBLIOGRAFÍA}

1. Riddell V. Thyroidectomy: prevention of bilateral recurrent nerve palsy. Results of identification of the nerve over 23 consecutive years (1946-69) with a description of an additional safety measure. Br J Surg. 1970;57(1):1-11.

2. Pardal-Refoyo JL, Ochoa-Sangrador C, Cuello-Azcárate JJ, Martín-Almendra MA. Rev Soc Otorrinolaringol Castilla Leon Cantab La Rioja. Precisión de la neuromonitorización en cirugía tiroidea. 2013;4(23):175-93. Disponible en: http://hdl.handle.net/10366/124525. [Citado el 9 de julio de 2016].

3. Henry LR, Abad JD, Stojadinovic A. The voice, not the nerve, is the functionally relevant endpoint. J Surg Oncol. 2012;106(8):1005-6.
4. Ochoa-Sangrador C. Evidencia y recomendación. Rev. ORL. 2016;7(2):67-71. Disponible

en:

http://dx.doi.org/10.14201/orl201672.14019. [Citado 9 de julio de 2016].

5. Sanabria Á, RamírezA. Economic analysis of routine neuromonitoring ofrecurrent laryngeal nerve in total thyroidectomy. Biomedica. 2015;35(3):363-71.

6. Pardal-Refoyo JL, Ochoa-Sangrador C. Bilateral recurrent laryngeal nerve injury in total thyroidectomy with or without intraoperative neuromonitoring.S ystematic review and meta-analysis. Acta Otorrinolaringol Esp. 2016;67(2):66-74.

7. Rulli F, Ambrogi V, Dionigi G, Amirhassankhani S, Mineo TC, Ottaviani $F$, et al. Meta-analysis of recurrent laryngeal nerve injury inthyroid surgery with or without intraoperative nerve monitoring. Acta Otorhinolaryngol Ital. 2014;34(4):223-9.

8. Pisanu A, Porceddu G, Podda M, Cois A, Uccheddu A. Systematic review with meta-analysis of studies comparing intraoperative neuromonitoring of recurrent laryngeal nerves versus visualization alone during thyroidectomy. J Surg Res. 2014;188(1):152-61

9. Zheng S, Xu Z, Wei Y, Zeng M, He J. Effect of intraoperative neuromonitoringon recurrent laryngeal nerve palsy rates after thyroid surgery-a meta-analysis. J Formos Med Assoc. 2013;112(8):463-72.

10. Sanabria A, Ramírez A, Kowalski LP, Silver CE, Shaha AR, Owen RP, et al. Neuromonitoring in thyroidectomy: a metaanalysis of effectiveness from randomized controlled trials. Eur Arch Otorhinolaryngol. 2013;270(8):2175-89.

11. Higgins TS, Gupta R, Ketcham AS, Sataloff RT, Wadsworth JT, Sinacori JT. Recurrent laryngeal nerve monitoring versus identification alone onpost-thyroidectomy true vocal fold palsy: a meta-analysis. Laryngoscope. 2011;121(5):1009-17. 